\title{
Simultaneous Exact Controllability of Wave Sound Propagations in Multilayer Media
}

\author{
Alexis Rodriguez Carranza, Luis. J. Caucha, Obidio Rubio Mercedes \\ National university of Trujillo, Peru.
}

Received: January 30, 2016 / Accepted: February 26, 2016 / Published: August 25, 2016.

\begin{abstract}
In this work we study one problem of mathematical interest for their applications in several topics in Applied Science. We study simultaneous controllability of a pair of systems which model the evolution of sound in a compressible flow considered as a transmission problem. We show the well posed of the problem. Furthermore provided appropriate conditions in the geometry of the domain are valid and suitable assumptions on the fluid, is possible to conduce the pair of systems to the equilibrium in a simultaneous way using only one control.
\end{abstract}

Key words: Controllability, wave sound, H.U.M

\section{Introduction}

In this work, we considered an equations system to describe an evolution of the wave sound or compressible fluids. A linear model well know is given by a system [7]

$$
\left\{\begin{array}{c}
\frac{\partial u}{\partial t}+\alpha \nabla p=0, \quad \text { in } \quad \Omega \times(0, T) \\
\frac{\partial p}{\partial t}+\beta \operatorname{div}(u)=0, \quad \text { in } \quad \Omega \times(0, T) \\
u . \eta=Q, \quad \text { in } \quad S_{0} \times(0, T) \\
p=0, \quad \text { in } \quad S_{1} \times(0, T) \\
u(x, 0)=u_{0}(x), p(x, 0)=p_{0}(x)
\end{array}\right.
$$

where $p=p(x, t) \quad$ is acoustic pressure, $u=\left(u_{1}, u_{2}, u_{3}\right)$ and $u_{j}=u_{j}(x, t)$ are fluid velocity field, $\alpha>0$ is the density of equilibrium and $\beta>0$ is the compressibility factor of fluid. Here $\Omega$ is an open subset of $I R^{3}$ with regularity boundary conditions $S_{0} \cup S_{1}=\partial \Omega \quad$ and $S_{0} \cap S_{1}=\varnothing$.

To solve the simultaneous controllability we considered a system given by

Corresponding author: Alexis Rodriguez Carranza, National university of Trujillo, Peru.

$$
\left\{\begin{array}{c}
\frac{\partial v}{\partial t}+\gamma \nabla q=0, \quad \text { in } \quad \Omega \times(0, T) \\
\frac{\partial q}{\partial t}+\tau \operatorname{div}(v)=0, \quad \text { in } \Omega \times(0, T) \\
q=P, \quad \text { in } \quad S_{0} \times(0, T) \\
q=0, \quad \text { in } \quad S_{1} \times(0, T) \\
v(x, 0)=v_{0}(x), q(x, 0)=q_{0}(x)
\end{array}\right.
$$

where $\gamma>0$ and $\tau>0 . Q$ and $P$ in (1) and (2), respectively; these are control functions. In 1986 , D.L. Russell [11] and J.L.Lions [8] proposed to solve a exact controllability problem for an evolution model, using only one control function. They called that problem as simultaneous controllability. The absences of dissipative effects as in (1) and (2), the problem present difficulties for the solution, see the examples [4], [5] and [8], where they perturbed the multipliers used for the controllability.

The problem of simultaneous controllability for the systems (1) and (2) is to take a control for both of system using only one control function, i.e., given $T>0$ any initial condition, $\left(u_{0}, p_{0}, v_{0}, q_{0}\right)$, and final $\left(\tilde{u}_{0}, \tilde{p}_{0}, \tilde{v}_{0}, \tilde{q}_{0}\right)$ in appropriate functional space, find $P(x, t)$ and $Q(x, t)$ such that

A solution $\{u, p, v, q\}$ of (1) and (2) satisfied in $T$ 


$$
(u(., T), p(., T), v(., T), q(., T))=\left(\tilde{u}_{0}, \tilde{p}_{0}, \tilde{v}_{0}, \tilde{q}_{0}\right)
$$

The control function, $P(x, t)$, for (2) was given in terms of $Q(x, t)$.

A method to solve the controllability problem is Hilbert Uniqueness Method (H.U.M) proposed by J.L.Lions, it is a construction of an appropriate structure for the Hilbert space in the initial conditions space.

These structure are connected by uniqueness properties. An important contribution to the controllability problems (1) and (2) were made by Kapitonov et. G. Perla Menzala [5]. In [5] the author answered positively for a simultaneous control and They showed that the control $P=-\frac{\beta}{\gamma} Q$ could be use to solve a problem. In this work we study a controllability problem of these systems with a perspective for applications as a problem of transmission; this is described below.

$m>1$ and $k=1,2, \ldots, m$. For each $k$, look at $b_{k}$ uan open and connected subset with regular boundary and such that $\bar{\sigma}_{1} \subseteq b_{k} \subseteq \sigma_{0}, \bar{b}_{k} \subseteq b_{k+1}$. place $\omega_{0}=b_{1} \backslash \bar{\sigma}_{1}, \Omega_{k}=b_{k+1} \backslash \bar{b}_{k}$, $k=1,2, \ldots, m-1$ e $\omega_{m}=\sigma_{0} \backslash \bar{b}_{m}$.
Given $\sigma_{0}$ and $\sigma_{1}$ open limited subset and connected in $i r^{3}$, with $\bar{\sigma}_{1} \subseteq \sigma_{0}$. Also $\omega=\sigma_{0} \backslash \bar{\sigma}_{1}$, we denoted $\partial \sigma_{0}=s_{0}, \partial \sigma_{1}=s_{1}$. And fixed an integer $m>1$ and $k=1,2, \ldots, m$. For each $k, b_{k}$ is an open subset and connected, with regularity in the boundary such that, $\bar{\sigma}_{1} \subseteq b_{k} \subseteq \sigma_{0}$, $\bar{b}_{k} \subseteq b_{k+1}$. We put $\omega_{0}=b_{1} \backslash \sigma_{1}, \omega_{k}=b_{k+1} \backslash \bar{b}_{k}$, $k=1,2, \ldots, m-1$ and $\omega_{m}=\sigma_{0} \backslash \bar{b}_{m}$. and, $\omega=\cup_{j=0}^{m} \omega_{j}$, for $i \neq j$, we take $\omega_{i} \cap \omega_{j}=\varnothing$ and $\partial \omega=s_{0} \cup s_{1}$. Examples for this decomposition is showed in Fig 1.

We need a solution defined by part on each sub domain; for that, we considered the systems (1) and (2) rewrite on sub domains $\Omega_{k}$, and

$$
\left\{\begin{array}{ccc}
\frac{\partial u^{k}}{\partial t}+\alpha^{k} \nabla p^{k}= & 0, & \text { in } \Omega_{k} \times(0, T) \\
\frac{\partial p^{k}}{\partial t}+\beta^{k} \operatorname{div}\left(u^{k}\right)= & 0, & \text { in } \Omega_{k} \times(0, T) \\
u^{k}(x, 0)= & u_{0}^{k}(x), & p^{k}(x, 0)=p_{0}^{k}(x)
\end{array}\right.
$$

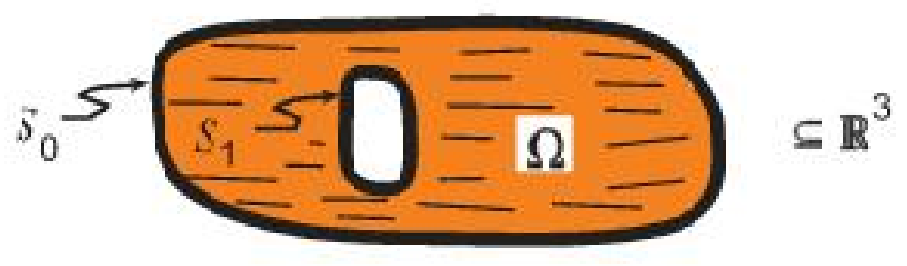

FIG. 1. $n=0$

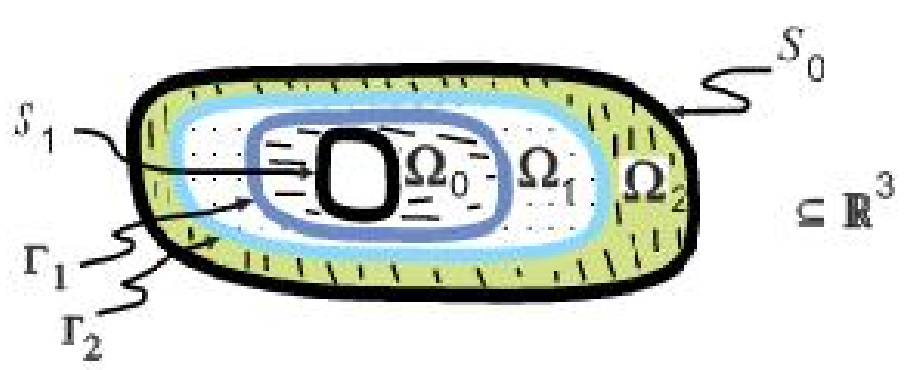

Fig. 1 Case $m=0$ and $m=3$. 


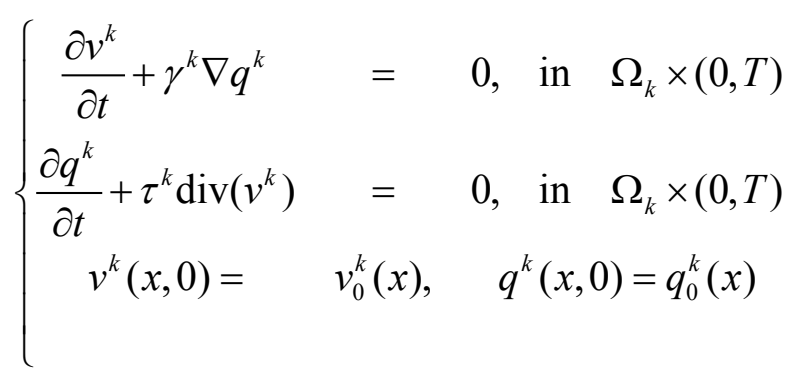

$$
k=0,1,2, \ldots, m .
$$

with boundary conditions (1) and (2). The interfaces of transmission conditions $\Gamma_{k}=\partial \Omega_{k}$, given by

$$
\begin{gathered}
\left\{\begin{array}{ccc}
\alpha^{k-1} p^{k-1} & = & \alpha^{k} p^{k} \\
\beta^{k-1}\left(u^{k-1} \cdot \eta\right) & = & \beta^{k}\left(u^{k} \cdot \eta\right) \\
k=2, \ldots, m, & (x, t) \in \Gamma_{k} \times(0, T)
\end{array}\right. \\
\left\{\begin{array}{ccc}
\gamma^{k-1} q^{k-1} & = & \gamma^{k} q^{k} \\
\tau^{k-1}\left(v^{k-1} \cdot \eta\right) & = & \tau^{k}\left(v^{k} . \eta\right) \\
k=2, \ldots, m, & (x, t) \in \Gamma_{k} \times(0, T)
\end{array}\right.
\end{gathered}
$$

for the systems (3) and (4), respectively.

The functions $\alpha^{k}, \beta^{k}, \gamma^{k}$ and $\tau^{k}$ are the restriction for the functions $\alpha, \beta, \gamma, \tau$ on the systems (1) and (2), we assumed that those functions were constant by parts, strictly positive and we lost the continuity only in $\Gamma_{k}, k=1,2, \ldots, m$.

The objective in this section is to get the estimation of

$$
\begin{aligned}
& \left(T-T_{0}\right) \sum_{k=0}^{m} \int_{\Omega_{k}}\left[\begin{array}{c}
\beta^{k}\left|u^{k}\right|^{2}+\alpha^{k}\left(p^{k}\right)^{2} \\
+\tau^{k}\left|v^{k}\right|^{2}+\gamma^{k}\left(q^{k}\right)^{2}
\end{array}\right] d x \\
& \leq C \int_{0}^{T} \int_{S_{0}}[\alpha p-\tau(v . \eta)]^{2} \frac{\partial h}{\partial \eta} d S_{0} d t .
\end{aligned}
$$

For some $T_{0}>0, C>0$ and $T>T_{0}$. The inequality (7) is named from an inequality of observation which is in the theorem 3 assuming geometrical properties on domain $\Omega$ and in the interfaces $\Gamma_{k}$. Such that, to prove (7) we assumed monotonicity conditions in the coefficients of the systems (3) and (4). The requirement necessary were found by Lions[8] in his study of transmission problem. Lagnese[4] used the same hypothesis to prove the result of controllability for a hyperbolic problem.

\section{Functional Spaces}

Given the Hilbert space $X_{1}=\left[L^{2}(\Omega)\right]^{3} \times\left[L^{2}(\Omega)\right]$, associate to (3). We define an scalar product in $X_{1}$, given by $(\tilde{u}, \tilde{p}),(u, p) \in X_{1}$, then:

$$
\langle(u, p),(\tilde{u}, \tilde{p})\rangle_{X_{1}}=\sum_{k=0}^{m} \int_{\Omega_{k}}\left\{\beta^{k} u^{k} \cdot \tilde{u}^{k}+\alpha^{k} p^{k} \tilde{p}^{k}\right\} d x
$$

Consequently, we considered $X_{2}=\left[L^{2}(\Omega)\right]^{3} \times\left[L^{2}(\Omega)\right]$ associate to (4). We define a scalar product in $X_{2}$, as $(\tilde{v}, \tilde{q}),(v, q) \in X_{2}$, then:

$$
\langle(v, q),(\tilde{v}, \tilde{q})\rangle_{X_{2}}=\sum_{k=0}^{m} \int_{\Omega_{k}}\left\{\tau^{k} v^{k} \cdot \tilde{v}^{k}+\gamma^{k} q^{k} \tilde{q}^{k}\right\} d x
$$

We have considered a total energy to the problem (3), (4), (5), (6) and the boundary conditions in (1), (2), as

$$
E(t)=\frac{1}{2} \sum_{k=0}^{m} \int_{\Omega_{k}}\left\{\begin{array}{l}
\beta_{k}\left|u^{k}\right|^{2}+\alpha^{k}\left(p^{k}\right)^{2}+ \\
\tau_{k}\left|v^{k}\right|^{2}+\gamma^{k}\left(q^{k}\right)^{2}
\end{array}\right\} d x
$$

Making a rigorously way for the interfaces conditions, we can see a lemma 1 ; for more details see Perla et al.[5].

Lemma 1. Given $\Omega$ bounded region in $I R^{3}$, with regularity in the boundary $\partial \Omega$. The application

$$
\begin{array}{ccc}
{\left[C^{1}(\bar{\Omega})\right]^{3}} & \rightarrow & C^{1}(\partial \Omega) \\
u=\left(u_{1}, u_{2}, u_{3}\right) & \rightarrow & u . \eta
\end{array}
$$

where $\eta=\eta(x)$ is as exterior unit normal vector in 
$x \in \partial \Omega$. We can extend by continuity application

$$
\tilde{H} \longrightarrow H^{-1 / 2}(\partial \Omega)
$$

where $\tilde{H}=\left\{u \in\left[L^{2}(\Omega)\right]^{3}, \quad \operatorname{div}(u) \in L^{2}(\Omega)\right\}$ and $H^{-1 / 2}(\partial \Omega)$ is dual space of $H^{1 / 2}(\partial \Omega)$

To simplify the notation we write $u^{k}$ as $u, \beta^{k}$ as $\beta$, the same way for all symbols in the region $\Omega_{k}$. by the lemma 1 is clearly that the spaces

$$
\begin{aligned}
& H_{1}=\left\{\begin{array}{l}
(u, p) \in X_{1}, \\
(-\alpha \nabla p,-\beta \operatorname{div}(u)) \in X_{1}
\end{array}\right\} \subseteq X_{1} \\
& H_{2}=\left\{\begin{array}{l}
(v, q) \in X_{2}, \\
(-\gamma \nabla q,-\tau \operatorname{div}(v)) \in X_{2}
\end{array}\right\} \subseteq X_{2}
\end{aligned}
$$

we can define the sub spaces:

$$
Z_{1}=\left\{\begin{array}{ll}
(u, p) \in H_{1}, & \\
\alpha^{k-1} p^{k-1}= & \alpha^{k} p^{k} \\
\beta^{k-1}\left(u^{k-1} \cdot \eta\right)= & \beta^{k}\left(u^{k} \cdot \eta\right), \operatorname{in} \Gamma_{k} \cdot \\
u . \eta=0 \in S_{0}, & p=0 \in S_{1}
\end{array}\right\}
$$

and,

$$
Z_{2}=\left\{\begin{array}{cc}
(v, q) \in H_{2}, & \\
\gamma^{k-1} q^{k-1}= & \gamma^{k} q^{k} \\
\tau^{k-1}\left(v^{k-1} \cdot \eta\right)= & \tau^{k}\left(v^{k} \cdot \eta\right) \quad \text { in } \Gamma_{k}, \\
q=0, & \text { in } \quad \partial \Omega=S_{0} \cup S_{1}
\end{array}\right\}
$$

Observe that $\left[C^{1}(\bar{\Omega})\right] \subset Z_{j}, \quad j=1,2$. Also $Z_{1}$ and $Z_{2}$ are dense in $X_{1}$ and $X_{2}$, respectively. Considering the bounded operator

$$
A_{j}: Z_{j}=D\left(A_{j}\right) \subseteq X_{j} \longrightarrow X_{j}
$$

defined as

Given

$$
(u, p) \in D\left(A_{1}\right)
$$

then,

$$
A_{1}(u, p)=(-\alpha \nabla p,-\beta \operatorname{div}(u))
$$

Given

$$
(v, q) \in D\left(A_{2}\right)
$$

then,

$$
A_{2}(v, q)=(-\gamma \nabla q,-\tau \operatorname{div}(v))
$$

Perla et al. [5] showed that operator $A_{1}$ is skew-adjoint, i.e, $A_{1}^{*}=-A_{1}$, the same result was proved for $A_{2}$. Using the Stone's theorem, we have proved that $A_{1}$ and $A_{2}$ generate infinitesimally a group of strongly continuous unit operators $\left\{U_{j}(t)\right\}_{t \in I R}$, in $X_{1}$ and $X_{2}$, respectively. Moreover, $U_{j}(t) w_{j}$ is strongly differentiable in relation to $t$ and for any $w_{j} \in D\left(A_{j}\right)$,

$$
\frac{d}{d t} U_{j}(t) w_{j}=A_{j} U(t) w_{j}
$$

\section{Inequality of Observability}

Using the multiplier's theory (see Komornik[3]), we make the proof. The multiplier was modified to get a good estimates in the boundary. These multiplier were used in several works. The invariant of the systems (1) and (2), in relations to dilatations groups in all variables, see [5]. Given $h: C(\Omega) \cap C^{1}(\bar{\Omega}) \longrightarrow I R$ an auxiliary function, it will be chosen in the next steps; and, given $(u, p) \in V_{1} \cap D\left(A_{1}\right)$ a solution of the system (1). Considering the multiplier given by:

$$
\left\{\begin{array}{l}
M_{1}=2\left(\alpha t p-u . \nabla h+\alpha \int_{0}^{t} p(x, s) d s\right) \\
M_{2}=2(\beta t u-p \nabla h) \\
M_{3}=2 \beta u
\end{array}\right.
$$

and $(u, p)$ solution of (1), we have the identities

$$
\begin{aligned}
& 0=M_{1}\left\{p_{t}+\beta \operatorname{div} u\right\}+M_{2} \cdot\left\{u_{t}+\alpha \nabla p\right\}+ \\
& M_{3} \cdot\left\{\int_{0}^{t}\left(u_{s}+\alpha \nabla p\right) d s\right\}
\end{aligned}
$$


The expression above, we make rewrite as

$$
0=\frac{\partial A}{\partial t}-\operatorname{div}(\vec{B})-J
$$

where

$$
\begin{aligned}
& A=t\left[\beta|u|^{2}+\alpha p^{2}\right]-2 p(u . \nabla h)+2 \alpha p \int_{0}^{t} p(x, s) d s-2 \beta u(x, 0) \cdot \int_{0}^{t} u(x, s) d s \\
& \vec{B}=-2 \alpha \beta t p u+\alpha p^{2} \nabla h-\beta|u|^{2} \nabla h+2 \beta(u . \nabla h) u-2 \alpha \beta\left(\int_{0}^{t} p(x, s) d s\right) u \\
& J=\beta(\Delta-1)|u|^{2}-2 \beta \sum_{i, j=1}^{3} \frac{\partial^{2} h}{\partial x_{i} \partial x_{j}} u_{i} u_{j}-\alpha(\Delta h-3) p^{2}
\end{aligned}
$$

We choosea function as:

$$
h(x)=\frac{1}{2}\left|x-x_{0}\right|^{2}+\delta_{0} \Phi(x)
$$

where $\mathrm{x}_{0} \in \sigma_{1}$ and $\Phi$ satisfy

$$
\left\{\begin{array}{l}
\Delta \Phi=1 \text { en } \Omega \\
\frac{\partial \Phi}{\partial \eta}=2 \frac{\operatorname{Vol}(\Omega)}{\operatorname{area}\left(S_{0}\right)}, \text { in, } S_{0} \\
\frac{\partial \Phi}{\partial \eta}=-\frac{\operatorname{Vol}(\Omega)}{\operatorname{area}\left(S_{1}\right)}, \text { in, } S_{1}
\end{array}\right.
$$

Is easy show that $\mu(\Omega) \leq \frac{2}{3}$

$$
\left\{\begin{aligned}
u_{0}^{k-1} \cdot \eta & =u_{0}^{k} \cdot \eta \quad \text { em } \quad \Gamma_{k} \\
u_{0}^{k-1} \times \eta & =u_{0}^{k} \times \eta \quad \text { em } \quad \Gamma_{k} \\
u_{0}^{k} & =\nabla l^{k}(x) \quad \text { em } \quad \Gamma_{k} \\
l^{k} & \in H^{2}\left(\Omega_{k}\right) \quad \text { e } \quad h=0 \quad \text { em } \quad S_{1}
\end{aligned}\right.
$$

Now, considered the hypothesis in the domain $\Omega$. Given $\delta_{0}>0$ such that, some $x_{0} \in \sigma_{1}$, we have

$$
\left\{\begin{array}{l}
\delta_{0}(1-\mu(\Omega))<1, \\
\left(x-x_{0}\right) \cdot \eta \geq-2 \delta_{0} \frac{\operatorname{Vol}(\Omega)}{\operatorname{area}\left(S_{0}\right)}, \quad \text { for } \quad x \in S_{0} \\
\left(x-x_{0}\right) \cdot \eta \leq \delta_{0} \frac{\operatorname{Vol}(\Omega)}{\operatorname{area}\left(S_{1}\right)}, \quad \text { for } \quad x \in S_{1} \\
\left(x-x_{0}\right) \cdot \eta+\delta_{0} \frac{\partial \Phi}{\partial \eta} \geq 0, \quad \forall x \in \Gamma_{k}, \quad k=1,2, \square, m \\
\alpha^{k-1} \leq \alpha^{k}
\end{array}\right.
$$

$$
\beta^{k-1} \leq \beta^{k}
$$

Lemma 1. Taking $\Phi$ as in (13), the geometry properties (15), and the hypothesis of monotony of coefficients (16) and the hypothesis (14); these were made for the initial condition. Then, $\exists C_{5}>0$, withindependence of $t, u, u_{0}, p_{0}$, such that

$$
\begin{aligned}
& \frac{T}{2}\left[1-\delta_{0}(1-\mu(\Omega))-2 C_{5}\right] \times \\
& \sum_{k=0}^{m} \int_{\Omega_{k}}\left[\beta^{k}\left|u^{k}\right|^{2}+\alpha^{k}\left(p^{k}\right)^{2}\right] d x \\
& \leq 2 \int_{0}^{T} \int_{S_{0}} \alpha p^{2} \frac{\partial h}{\partial \eta} d S_{0} d t .
\end{aligned}
$$

The same manner, we obtain the inequality of observability for the system (2)-(4) with their interface conditions and the monotonicity of the coefficients, given by:

$$
\begin{aligned}
& \gamma^{k-1} \leq \gamma^{k} \\
& \tau^{k-1} \leq \tau^{k}
\end{aligned}
$$

Theorem 2. Assuming $\Phi$ as in (13), the monotonicity for the coefficients (17) and the hypothesis of the theorem 1 with $h(x)=\frac{1}{2}\left|x-x_{0}\right|^{2}+\delta_{0} \Phi(x) \quad$ and $\left(v_{0}, q_{0}\right) \in V_{2} \cap D\left(A_{2}\right) \quad, \quad v_{0}^{k}=\nabla m^{k} \quad, \quad$ with $m^{k} \in H^{2}\left(\Omega_{k}\right), m=0$ in $S_{1}$. Then, there is a constant $C_{6}>0$, with independence of $t, v_{0}, q_{0}$ such that 


$$
\begin{aligned}
& T\left[1-\delta_{0}(1-\mu(\Omega))\right] \sum_{k=0}^{m} \int_{\Omega_{k}}\left[\tau^{k}\left|v^{k}\right|^{2}+\gamma^{k}\left(q^{k}\right)^{2}\right] d x- \\
& 2 C_{6} \sum_{k=0}^{m} \int_{\Omega_{k}}\left[\tau^{k}\left|v^{k}\right|^{2}+\gamma^{k}\left(q^{k}\right)^{2}\right] d x \\
& \leq 2 \int_{0}^{T} \int_{S_{0}} \tau|v \cdot \eta|^{2} \frac{\partial h}{\partial \eta} d S_{0} d t .
\end{aligned}
$$

Theorem 3. Assuming the hypothesis of the theorem 1, 2. Moreover, we suppose that :

$$
\begin{aligned}
& \alpha^{k} \beta^{k}=\gamma^{k} \tau^{k} \\
& \beta^{k-1} \tau^{k}=\beta^{k} \tau^{k-1} .
\end{aligned}
$$

Then, there is a positive constant $C>0$ such that

$$
\begin{gathered}
\left(T-T_{0}\right) \sum_{k=0}^{m} \int_{\Omega_{k}}\left[\begin{array}{c}
\beta^{k}\left|u^{k}\right|^{2}+\alpha^{k}\left(p^{k}\right)^{2} \\
+\tau^{k}\left|v^{k}\right|^{2}+\gamma^{k}\left(q^{k}\right)^{2}
\end{array}\right] d x \\
\leq C \int_{0}^{T} \int_{S_{0}}[\alpha p-\tau(v \cdot \eta)]^{2} \frac{\partial h}{\partial \eta} d S_{0} d t . \\
\forall T>\max \left\{1, \frac{C_{5}+C_{6}}{1-\delta_{0}(1-\mu(\Omega))}\right\} .
\end{gathered}
$$

\section{Exact Controllability}

Given $G_{1}=\left(w_{0}, k_{0}\right) \quad$ and $\quad G_{2}=\left(m_{0}, l_{0}\right)$ arbitrary elements of $Y$. We denote by

$$
\begin{aligned}
& (w(x, t), k(x, t))=U_{1}(t)\left(w_{0}, k_{0}\right) \\
& (m(x, t), l(x, t))=U_{2}(t)\left(m_{0}, l_{0}\right)
\end{aligned}
$$

consider the following functions

$$
\begin{aligned}
& Q=\beta(\alpha k(x, t)-\tau m(x, t) \cdot \eta) \\
& P=-\frac{\beta}{\gamma} Q
\end{aligned}
$$

and given $(u, p)$ and $(v, q)$ the solution of (3) and (4), these are null in the instant $T,\left(T>T_{0}\right)$ and the contour conditions (19).

Considering the map

$$
\begin{array}{ll}
\wedge: & Y \longrightarrow Y^{\prime} \\
& \left(G_{1}, G_{2}\right) \longrightarrow \wedge\left(G_{1}, G_{2}\right)=\left.(u, p, v, q)\right|_{t=0}
\end{array}
$$

Using (?) in $t=0$ and substituting $P$ and $Q$ given by (19), we have

$$
\begin{aligned}
& \left\langle\wedge\left(G_{1}, G_{2}\right),\left(\tilde{u}_{0}, \tilde{p}_{0}, \tilde{v}_{0}, \tilde{q}_{0}\right)\right\rangle_{X} \\
& =\int_{0}^{T} \int_{\Gamma_{0}}[\alpha \beta Q \tilde{p}+\gamma \tau P \tilde{v} . \eta] \\
& =\int_{0}^{T} \int_{\Gamma_{0}}(\gamma k-\tau m . \eta)(\alpha \tilde{p}-\tau \tilde{v} . \eta) \\
& =\left\langle\left(G_{1}, G_{2}\right),\left(\tilde{u}_{0}, \tilde{p}_{0}, \tilde{v}_{0}, \tilde{q}_{0}\right)\right\rangle_{Y}
\end{aligned}
$$

of (20), we can conclude that $\wedge$ is an isomorphism of $Y$ in $Y^{\prime}$. We proved following theorem

Theorem 1. Assuming the hypothesis of theorem 3. If given $T>T_{0}$ and initial condition $\left(u_{0}, p_{0}, v_{0}, q_{0}\right) \in Y^{\prime}$ the problem (1), (2), (5), (5). Then, there is exist an control $Q(x, t) \in C\left(0, T ; L^{2}\left(\Gamma_{0}\right)\right) \quad$ such that the corresponding solution $(u, p, v, q)$ with the boundary condition

$$
\left\{\begin{array}{l}
u . \eta=Q, \text { in } S_{0} \times(0, T) \\
p=0, \text { in } S_{1} \times(0, T) \\
u(x, 0)=u_{0}(x), p(x, 0)=p_{0}(x)
\end{array}\right.
$$

and

$$
\left\{\begin{array}{l}
q=P, \quad \text { in } S_{0} \times(0, T) \\
q=0, \quad \text { in } S_{1} \times(0, T) \\
v(x, 0)=v_{0}(x), q(x, 0)=q_{0}(x)
\end{array}\right.
$$

with $P=-\beta \gamma^{-1} Q$, satisfy, for $x \in \Omega$

$$
\left\{\begin{array}{l}
u(x, T)=0 \\
p(x, T)=0 \\
v(x, T)=0 \\
q(x, T)=0
\end{array}\right.
$$

\section{Acknowledgment}

We thank to professors G. Perla Menzala for the suggestions in this article.

\section{References}

[1] R. A. Adams, Sobolev Spaces, Academic Press, New York, 1975.

[2] J. M. Coron, Control and nonlinearity, Mathematical Survey and Monographs 2009. 
[3] V. Komornik, Exact Controllability and Stabilization. The multiplier method, RAM:Research in Applied Mathematics. Masson, Paris; Jhon Wiley and Sons, Chichester, 1994.

[4] J. E. Lagnese, Boundary controllability in problems of transmission for a class of second order hyperbolic systems, ESAIM Control Optim. Calc Var., 2 (1997), 343-357

[5] B.V. Kapitonov, and G. Perla , Simultaneous exact controllability for a pair of systems of evolution of sound in a compressible fluid, Journal of Mathematics and System Science 3 (2013) 655-658

[6] B.V. Kapitonov, and G. Perla, Boundary Stabilization and a problem of transmission for a system of propagation of sound, Funkcialaj Ekvacioj, 49 (2006), 107-132.
[7] L.D. Landau and E.M. Lifshitz, Fluid Mechanics Volume 6 of Course of Theorical Physics Pergamon Press, Oxford, New York, Paris (1960)

[8] J. L. Lions, Extact controllability, Stabilization and perturbations for distributed system, SIAM Review 30 (1988), 1-68.

[9] A. Pazy, Semigroup of Linear Operator and Application to Partial Differential equations, Springer-Verlag, New York, Berlin, 1983.

[10] D. L. Russell, A unified boundary controllability theory for hyperbolic and parabolic partial differential equations, Studies in Appl. Math. 52 (1973), 189-211.

[11] D. L. Russell, Controllability and Stabilizability theory for linear partial differential equations: recent progress an open questions, SIAM Review 20 (4) (1978), 639-739. 